\title{
Simple Control Law Structure for the Control of Airplanes by Means of Their Engines
}

\author{
Nicolas Fezans
}

\begin{abstract}
In this paper a simple control law structure is presented for the control of airplanes using only the engines' thrust. For the design of such a propulsion controlled aircraft control law, the approach followed in this work is to look for the right level of performance in order to avoid both excessive engines activity and reduction of robustness properties. Another goal is to keep the control law and its tuning as simple as possible: for this a control law structure whose terms can easily be interpreted is proposed. The capability of the proposed control law to permit safe landing was shown by simulator tests as well as flight tests.
\end{abstract}

\section{Introduction}

One of the objectives of the research conducted at the DLR (German Aerospace Center) Institute of Flight Systems is to improve the safety of all types of aircraft. This objective takes a central part in many projects concerning human-machine interaction (e.g. situation awareness, training of pilots), detection of adverse conditions (e.g. gust, wake-vortices), fault detection and isolation, and reconfiguration of control laws.

In the past, several incidents and accidents were caused by partial or total loss of aircraft primary control systems. Even though a total loss of aircraft primary control systems is theoretically extremely rare, it did happen several times [1] with an estimate of more than 1200 casualties made in 1996 [2]. The fact that such events are extremely rare must be demonstrated during the certification process. Indeed, looking at the past as well as at accidents that happened more recently (e.g. DHL A300 in Baghdad in 2003), it appears clearly that the loss of primary control devices is generally a consequence of other failures with some complex relations between

Nicolas Fezans

DLR (German Aerospace Center) Institute of Flight Systems, Lilienthalplatz 7, 38108 Braunschweig, Germany, e-mail: nicolas.fezans@dlr.de 
them and in some cases related to maintenance errors or to external causes. The interactions between the faults and failures in such systems can be extremely complex. The author's point of view is that even with very serious work on safety analysis a significant number of elements of the fault tree of such complex systems will be omitted. The level of redundancy and the extensive use of computers and real tests in the design of modern aircraft allowed to reach very high safety standards. However, human errors in the maintenance or the design of the aircraft can still happen as well as adverse weather conditions, wake vortex encounters, or terrorist actions. When technically and economically possible, design and implementation of emergency systems capable of dealing with a wide spectrum of improbable situations should be made. The propulsion-based emergency control law presented in this paper is worth being integrated in modern transport aircraft as such an emergency system.

Several studies have been conducted at NASA in the 1990's to prove the feasibility of controlling the aircraft and landing using only the engines [1, 2, 3]. It appeared clearly at that time that a system assisting the pilot is required and that with such a system probability of casualties would drastically be reduced. Implementation of such a system in aircraft equipped with an autothrottle would not require any new hardware (and thus there would be no increase of weight) and for many modern aircraft only software modifications would be required to include this new functionality. However, more than 15 years later still no civil transport aircraft possesses such an emergency system. Even aircraft that were entirely designed after this technology was demonstrated in flight are not equipped!

In this paper, the case of total loss of primary control devices is considered. For such a deteriorated airplane a control law based on engine thrust is designed with the approach of keeping a very simple structure and using the lowest gains possible. Longitudinal control is achieved using symmetrical variations of thrust and lateral control using asymmetrical variations. This control strategy is similar to the one studied at NASA in the 1990's under the name of "Propulsion Controlled Aircraft" (PCA). As the name PCA properly describes the content of the work presented in this paper, it will also be used. At the DLR Institute of Flight Systems, experiments on Fault-Tolerant Control (FTC) are made using the ATTAS research aircraft [4, $5,6]$ : some of them leading to the control of the ATTAS using only the engines. In particular, approaches based on Model Predictive Control (MPC) were tested with the aim of reaching very high performance by taking directly into account the limitations of the airplane and in particular of its actuators and engines. In the current work a low-gain approach is preferred.

The paper is organized as follows: Section 2 presents the motivation for a structured control law with a low-gain tuning. Section 3 discusses the properties of the aircraft with PCA control law that are required to permit safe landings. Section 4 presents the control law: its structure, tuning, and protections. Section 5 presents a summary of the results obtained during a flight test that took place in November 2009 with the presented propulsion-based control law. 


\section{Motivation for a Structured Control Law with a Low-Gain Tuning}

Failures are not always extremely severe and in some cases it makes sense to try to integrate the deteriorated aircraft into the regular air traffic. The failures leading to the use of a control law based only on the engines are very severe. In such cases, an emergency would be declared and the real objective for the design of an emergency system capable of controlling the aircraft by means of the engines is to permit an acceptable landing. An acceptable landing for such an emergency system is, to the author's mind, a landing avoiding both the loss of human lifes and major injuries. Relatively low vertical speed and normal attitude (slightly positive pitch, small roll) are major criteria for such a landing.

Besides, in such a severe failure case, quite strong differences in terms of dynamical behavior between a nominal model of the airplane and the real deteriorated airplane can be expected. For instance, the Boeing 747 of the Japan Airlines flight 123 had lost almost the entire vertical stabilizer and a significant part of the left wing was missing in the case of the Airbus A300 of DHL in Baghdad. These differences lead to the need for strong robustness properties of the system, either by intrinsic robustness of the control law or by its adaptation. The robustness analysis of airplanes with a propulsion-based control law is challenging due to the slow and highly nonlinear responses of the engines. Their dynamics result from a combination of saturations, nonlinear dynamics and state-dependent rate-limiters. The use of typical robustness metrics for design purposes in such a case is very difficult. For this reason, it has been decided to focus in this work on the definition of a simple control law whose components can easily been interpreted. Thus, the approach here is to compensate the lack of practically usable rigorous mathematical tools for such problems by the use of physical interpretations. Precise evaluations of the obtained robustness properties could also be made by means of Monte-Carlo methods but have not yet been considered in this study. The only evaluations that have been made until now rely on several hundreds of simulations. It should be noticed that stability of the controller-augmented airplane is not required as pilots will be part of the closed-loop. Moreover, a stability criterion does not permit to estimate whether a pilot is really able to reach the airport and land or not.

However, to the best of the author's knowledge no classical handling qualities criterion is applicable to a propulsion controlled aircraft, therefore there is a need for a new evaluation of handling qualities for such an airplane. An objective of this research is thus to understand which design criteria are really important with pilots in the loop and which criteria can be withdrawn. The purpose of this paper is not to address this question, but to present the simple control law structure that has been designed in order to ease future simulator and flight-test studies that will address it. With an adequate tuning of this control law the pilots must be able to land successfully in a wide spectrum of situations. Later on, once the required performance criteria for such a propulsion controlled aircraft will be properly defined and understood, the design and the validation of such an emergency system will be eased. 
A single set of parameters has been used on the entire flight domain and for all configurations. The only scheduling made takes place in the inner loop which controls the engines: the proportional gain depends on the rotational speed of the fan (N1), see section 4.5. Such a choice would often be suboptimal, in particular if we want to force the closed-loop to exhibit some defined behavior. But for such a system the exact behavior of the controller-augmented aircraft is not very important: what matters is that pilots succeed in controlling it and finally succeed in landing the airplane. In our experiments and in the work presented here this single set of parameters with a low-gain tuning allowed pilots to control the aircraft and to follow both glideslope and localizer signals with a good precision. This shows that both high-gain solutions and control strategies based on reference models are not required for this application.

\section{Requirements}

In this section, the main requirements for successful approach and landing by means of a PCA system are discussed with focus on how desirable they are, how difficult it will be to reach them, and which trade-off between the performance criteria should be made. Obviously, classical handling qualities criteria are not applicable for an aircraft having a propulsion-based control law.

\subsection{Longitudinal Control}

The longitudinal motion is mainly composed of the phugoid mode and the shortperiod mode. The period of the phugoid is generally between 30 and 60 seconds for transport airplanes. The frequency of the short-period mode depends on the aircraft and its center of gravity location, but would typically lie between 1.5 and $3 \mathrm{rad} / \mathrm{s}$.

Increasing the total thrust of the engines leads to an increase of the energy rate of the aircraft. To really know the effect of this additional thrust on the movement of the aircraft, the pitch equation as well as the aerodynamics and mass characteristics of the aircraft must be known. For typical configurations, a simplified reasoning can be expressed as follows: a constant additional total thrust $\Delta T_{t}=\sum_{i} \Delta T_{i}>0$ leads to a positive variation of the flight path angle $\gamma$ and vice-versa, i.e. $\Delta T_{i}>0 \Rightarrow$ $\Delta \gamma(t \rightarrow \infty)>0$ and $\Delta T_{i}<0 \Rightarrow \Delta \gamma(t \rightarrow \infty)<0$. This makes it possible to control the trajectory of the aircraft in the vertical plane.

With the typical frequencies and damping ratios of the short-period mode and of the phugoid as well as the typical dynamics of engines, no real challenge is expected in designing and tuning a control law assisting the pilots in the control of the flight path angle. Such a control law will basically consist of controlling the phugoid (acceptable response time, good damping, and no static error on the flight path) while avoiding unnecessary excitation of the short-period mode. 


\subsection{Lateral Control}

The lateral dynamics of an aircraft are composed of:

- the Dutch roll mode exhibiting a pair of complex conjugate and stable poles with very low damping,

- the roll mode which is aperiodic and stable,

- the spiral mode which is slow and quite often slightly unstable.

As for the short-period mode, the Dutch roll mode and the roll mode are generally too fast to be significantly modified by means of the engines, in particular in the lowthrust domain that will generally be required for descent and approach. However, a control law based on thrust can easily modify the spiral mode in order to ease its control by a human pilot. For this, the coupling between yaw and roll is used: the pilot controls only the roll motion and the control law generates a yaw motion by means of asymmetric thrust allowing to get the induced roll corresponding to the pilot's commands. In previous studies PCA control laws were designed to follow a reference bank angle $\phi_{\text {ref }}$ that was provided by the pilot. During the current research activities several other possibilities have been investigated in the ATTAS ground simulator. In particular a rate-command attitude-hold and a combination of roll rate and bank angle commands are being tested. They are both based on the control law presented hereafter: the difference is the way pilots provide the references to the system.

Some reasonable goals for the lateral part of the control law are: to permit enough maneuverability, to reduce pilot workload by damping the lateral dynamics, and to ensure acceptable disturbance rejection without any action of the pilot.

\section{Propulsion-Based Control Law}

This section presents the propulsion-based control law that was used for the simulator tests and flight tests that are shown in section 5. The global structure of the control law is first presented (section 4.1). After that all elements constituing this structure are presented separately in sections $4.2-4.5$. The easiness of the physical interpretation of each component will appear clearly.

\subsection{Global Control Structure}

The global control structure is presented in figure 1. This structure is based on a cascade control strategy with inner loops controlling the engines through commands in terms of Power Lever Angle (PLA) and outer loops controlling the longitudinal and lateral motions through symmetric and asymmetric thrust. The inner loops have a crossfeeding in case of saturation as detailed in section 4.5 (see signals PLA $_{s a t} \mathrm{~L}$ 
and PLA $_{s a t} \mathrm{R}$ ). A block labeled "Mixing priorities \& Protections" connects the outer loops to the inner loops by allocating longitudinal $\left(\overline{\mathrm{N}}_{c m d}\right)$ and lateral $\left(\Delta \mathrm{N} 1_{c m d}\right)$ control actions to the two engines while satifying the limits for each engine. This leads to the two references $\mathrm{N} 1 \mathrm{~L}_{r e f}$ and $\mathrm{N} 1 \mathrm{R}_{r e f}$ that are provided to the inner loops. Although this does not appear very explicitly in figure 1 the "Mixing priorities \& Protections" block also connects the two outer loops by means of the antiwindup feedback signals $\overline{\mathrm{N}}_{\text {sat }}$ and $\Delta \mathrm{N} 1_{\text {sat }}$. This particular point is more detailed in section 4.4.

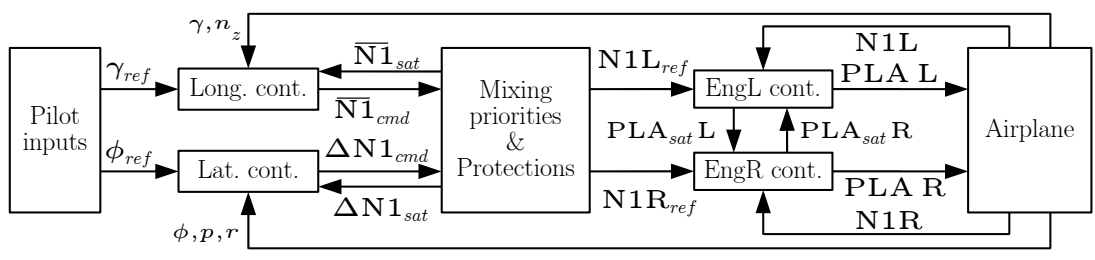

Fig. 1: Global architecture of the propulsion-based control law

On one side imposing a particular structure on the controller generally restricts the maximum achievable performance and robustness. On the other side it allows to guarantee both, the physical meaning of the controller's parameters and the simplicity of the future implementation on on-board computers. In this work the trade-off between reachable performance, engine activity, and robustness is investigated under the assumption that a simple and comprehensible structure is required. Using such a structure is therefore not a choice made during this work but a constraint directly included in the addressed problem itself.

\subsection{Longitudinal Controller}

The longitudinal controller is presented in figure 2a. It uses a PID structure with a filter $\left(K_{n_{z}}(s)\right)$ on the derivative part and two feedforward elements: a dynamical one $K_{F F \int \gamma}(s)$ and a static one $K_{F F \gamma}$. The filter $K_{n_{z}}(s)$ is required to remove the medium and high-frequencies measured by the accelerometer and therefore remove undesirable control activity due to gusts and turbulence. As the phugoid mode is very slow with respect to the typical frequency content of these types of atmospheric disturbances, a simple first-order low-pass filter with a bandwidth higher than the frequency of the phugoid mode was used.

To get the desired reference tracking of the flight path angle $\gamma$, the static gain $K_{F F \int \gamma}(\omega=0)$ must be equal to 1 . The degrees of freedom provided by these transfer functions allow seperate tuning of the reference tracking dynamics and of the disturbance rejection properties. For instance, defining $K_{F F} \int \gamma(s)$ as a low-pass filter permits to use a relatively high value for $K_{\int \gamma}$ without getting high overshoots of 
the reference tracking response and without requiring high values of the derivative gain. Such a tuning is interesting because it gives the pilot a control input that is not excessively sensible in the medium to high frequency domain while permitting quite efficient rejections of disturbances by the feedback without any action of the pilot. As the obtained performance was already satisfying with a simple gain for $K_{F F} \gamma$, this part of the feedforward has been kept static. If finer tuning of the reference tracking response is required some additional degrees of freedom could be obtained by using a transfer function instead of the $K_{F F} \gamma$ gain.

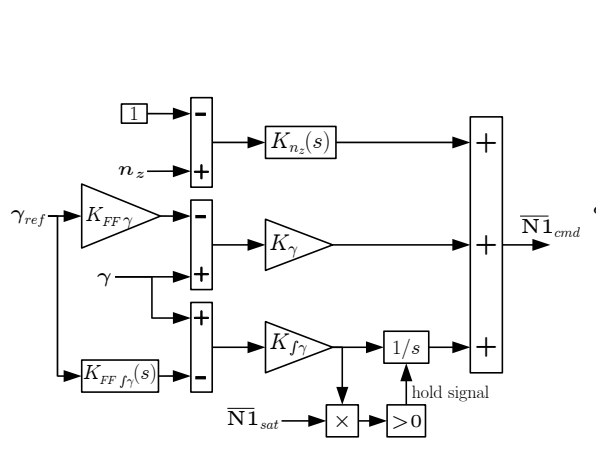

(a) Longitudinal: $\gamma$-mode

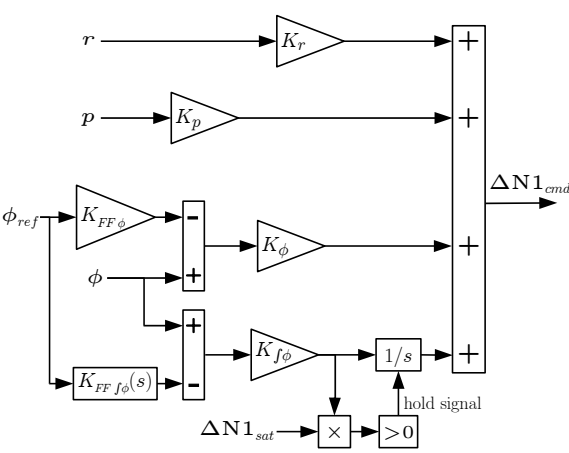

(b) Lateral: $\phi$-mode

Fig. 2: Longitudinal and lateral controllers

\subsection{Lateral Controller}

The lateral controller is based on the same principle as the longitudinal controller and therefore has a similar structure. However, the types of dynamics to be controlled by these two controllers are quite different. In the longitudinal case, the static response to a symmetric variation of thrust is a variation of the flight path angle $\gamma$, which is directly the variable that must be regulated. In the lateral case, the static response to an asymmetric variation of thrust is a variation of the sideslip angle $\beta$, which is related to the second time derivative of the regulated variable $\phi$ (bank angle).

This is a logical consequence of the fact that there is no roll authority and roll is now controlled by means of the yaw-roll coupling. Therefore, the chosen controller structure (figure $2 \mathrm{~b}$ ) is not only based on the bank angle $\phi$ and the roll rate $p$, but also on the yaw rate $r$. Feedback based on the sideslip angle $\beta$ would probably also help getting good closed-loop properties, but $\beta$ is generally not used in flight control systems for various practical reasons. This fact has been taken as a constraint and is not discussed. The feedback of $p$ and $r$ should ideally be restricted to high frequen- 
cies in order to avoid counteracting normal turns. Indeed the feedback $K_{\int \phi}$ ensures rejection of such side effects and simple gains $K_{p}$ and $K_{r}$ can be taken instead of some high-pass filters.

During the landing task, the required corrections for the lateral movement are much more demanding than for the longitudinal motion. This leads to a more difficult tuning of the lateral controller. The balance between good enough handling qualities and high activity of engines is more difficult to find, in particular when considering uncertainties of engines' dynamics. As mentioned in the introduction, the analysis tools for this type of nonlinear system are difficult to put into practice and with a pilot in the loop stability is neither a sufficient nor a required condition. Thanks to the physical interpretations of each part of the controller, a good set of parameters can be found in a short time. However, a more systematic and rigorous methodology would be desirable.

\subsection{Mixing Priorities and Outer Loop Protections}

Authority on both longitudinal and lateral movements is severely restricted. The same actuators (i.e. engines) are used for both and thus some mixing priorities should be defined. As the controlled dynamics of the lateral motion are faster than the controlled dynamics of the longitudinal motion, it seems logical to give priority to the lateral control action over the longitudinal control actions in the case both cannot be satisfied simultaneously. Moreover, a poor control of the spiral mode will strongly disturb the control of the flight path angle, whereas the opposite coupling can be neglected in most cases (e.g. whithout stall, overspeed, etc.). This priority can be implemented as shown in figure 3 where the absolute value of the lateral control signal is used in the definition of limits applied to the longitudinal control signal. This permits to ensure that the sum of both control signals will respect the constraints of each engine and to give priority to the thrust difference between them over the mean thrust.

In figure 3 , signals $\overline{\mathrm{N}}_{\text {sat }}$ and $\Delta \mathrm{N} 1_{\text {sat }}$ are respectively the differences between input and output of longitudinal and lateral control saturations. They are used for the "integrator hold" antiwindup strategy that is implemented in both controllers (figures 2a-2b). After giving priority to the thrust difference over the mean thrust, the signs of these signals are compared to the sign of the signals entering the integrators in figures $2 \mathrm{a}$ and $2 \mathrm{~b}$. If their signs are identical, then the corresponding integrator would be winding and this is prevented by holding the integrator at its previous value. This way of preventing integrator windup does not allow to desaturate the command in order to keep some authority, but in the considered application there is no need for a more complex antiwindup strategy. 


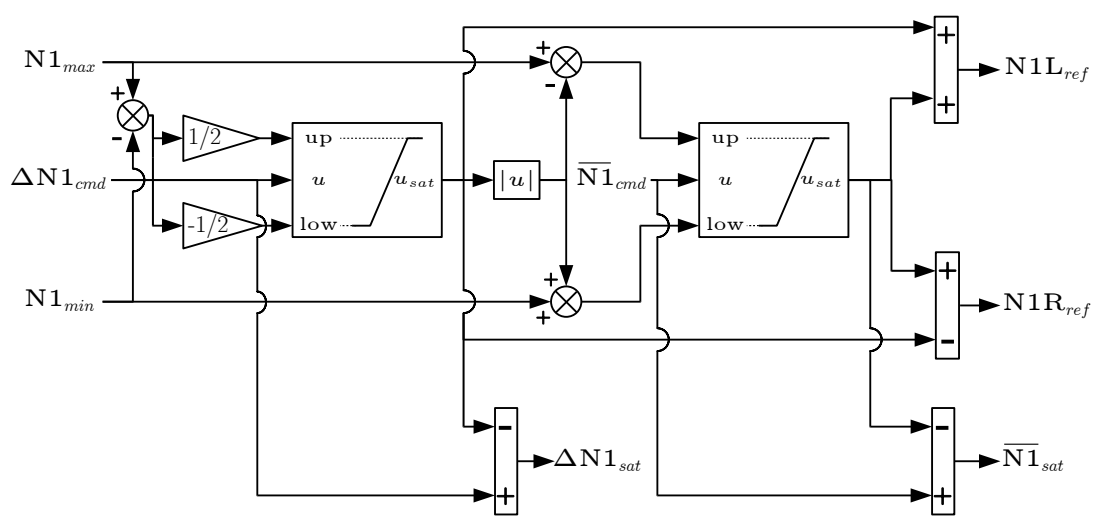

Fig. 3: Mixing priorities and outer-loops protections

\subsection{Inner Loops for Engines Control}

The structure of the inner loop controllers is depicted in figure 4. In this figure the controller of the left engine is shown: the letters $\mathrm{L}$ and $\mathrm{R}$ are use to distinguish left and right. For the right engine, the letters $\mathrm{L}$ and $\mathrm{R}$ must be inverted in this figure. The controller is basically a PI controller with a static feedforward on the proportional part and an "integrator hold" antiwindup strategy. In addition, the saturation signal of the other engine (here PLA $\mathrm{A}_{s a t} \mathrm{R}$ ) is crossfed in the output in accordance with the priorities defined in the previous section.

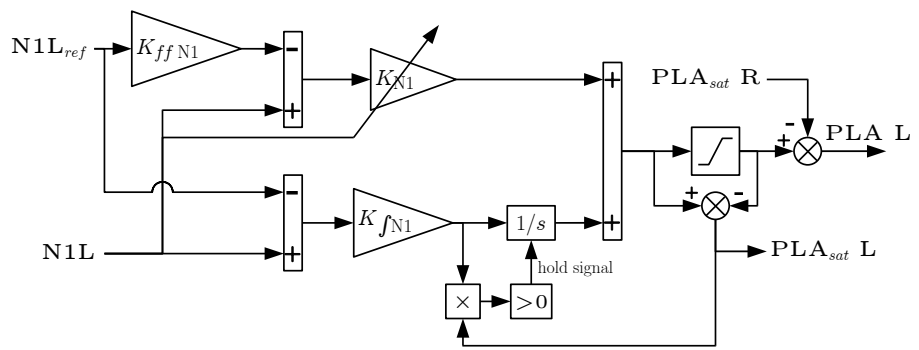

Fig. 4: Inner loop for the left engine control. Invert $\mathrm{L}$ and $\mathrm{R}$ for the right engine.

During our tests (both in simulator and in flight), the aforementioned antiwindup and crossfeed in these inner loops did not appear to be absolutely required as outer loop gains were intentionally chosen relatively low. For more performance-driven tuning or in the presence of uncertainties these elements are likely to be of the greatest importance. As they result in negligible increases of controller complexity, they should be kept for all implementations of such controllers. 
Note that in our applications, the parameters of these loops were systematically taken equal for both engines even though significant differences of the left and right engines were known and can be observed at medium to high frequencies (with respect to closed-loop bandwidth). Note that engines may also differ significantly during their life and the control law must be able to deal with such variations. This can be achieved through the robustness of the control law or by means of adaptive techniques. Adaptive techniques have not been considered at all during this work.

\section{Summary of Flight Test Results}

After several simulator tests of the system, the system was tested on Nov. 20, 2009 with the DLR ATTAS (VFW-614) research airplane. Goal of this flight test was to verify and test the handling qualities provided by this propulsion-based control law for navigation, ILS intercept, approach, and go-around. During previous simulator tests pilots were asked to rate several possible tuning options for the control law parameters and to express their preferences. These preferences were also put into perspective with their foreseeable drawbacks in terms of robustness, disturbance rejection properties, engine activity. During this analysis, rough assessments of the closed loop at 11 points of the flight domain were made. The considered points are based on 3 different flap configurations, 4 different speeds, 3 different altitudes, and landing gear extended or retracted (note that not all combinations of these parameters were considered). A $200 \mathrm{~ms}$ delay was introduced at engine inputs and for each flight condition the frequency of the Dutch roll of the ATTAS model was artificially shifted until the closed loop was destabilized. Besides, simulations with demanding inputs (e.g. successions of maximal stick inputs at various frequencies around the bandwidth of the stick to output transfer function) as well as simulations with initial conditions were performed with the aim of analyzing both the input-output behavior (including its nonlinearities) and the internal dynamics of the closed loop. The objective was not to compute robustness margins, but rather to obtain a qualitative evaluation of each set of parameters regarding robustness and disturbance rejection properties. More complete and precise evaluations will be performed later for validation purposes and not for design purposes. This analysis lead to test two controller parameter sets (called tuning A and tuning B hereafter) during this flight, even though a clear preference for tuning A had already been identified before the flight. It corresponds to a faster but less damped behavior for the lateral motion.

Weather was sunny and cloudless. Wind at $2500 \mathrm{ft}$ was 40 to $50 \mathrm{kt}$ from $220^{\circ}$ and wind on ground was $5 \mathrm{kt}$ from south-east. Light turbulence. After take-off and activation of the experimental systems, some maneuvers were made by the test pilot in order to check the dynamical behavior of the aircraft with this control law and to provide data with strong enough inputs for future analysis. A short sequence of these maneuvers is shown in figure 5. It shows how well the references were followed as well as how the deviations caused by the very light turbulence are rejected in spite 


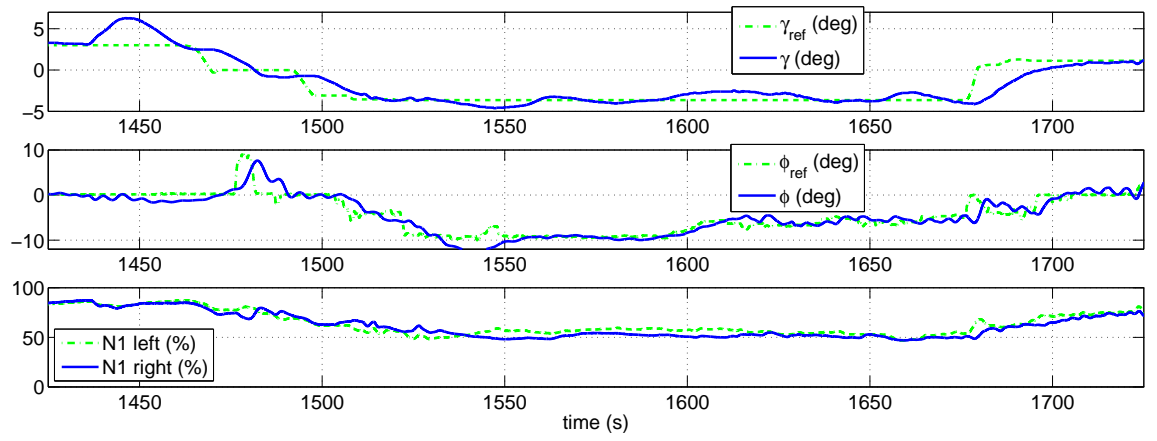

Fig. 5: Reference tracking with the propulsion-based control law.

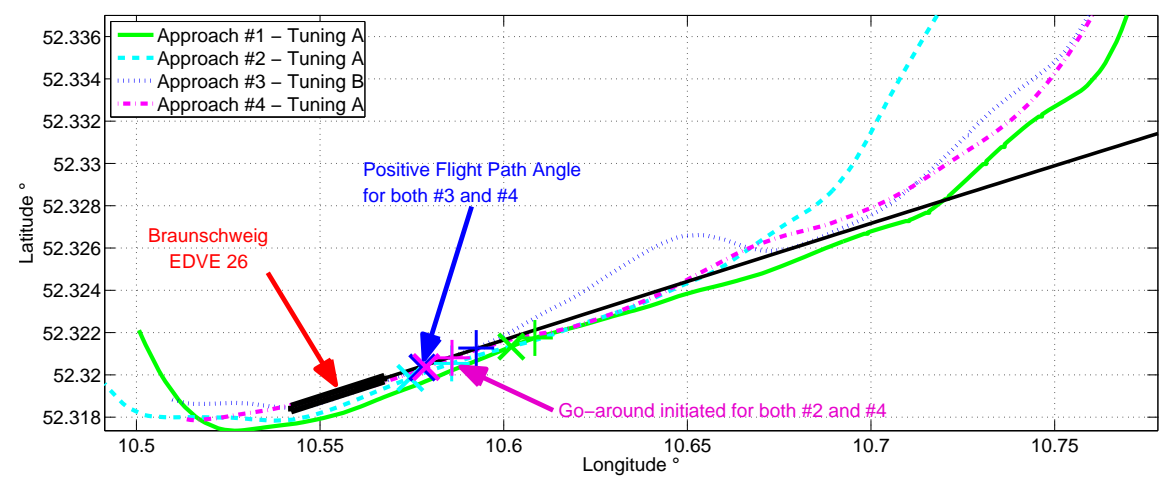

Fig. 6: Ground tracks of approaches performed with the PCA control law.

of low engine activity. After that the entire sequence "navigation, ILS intercept, approach, and go-around" has been flown four times.

The preference for tuning A was rapidly confirmed before attempting to land and therefore three of the four "intercept, approach, and go-around" sequences flown were performed using the tuning $\mathrm{A}$. The ground tracks of the sequences are shown in figure 6 . The longitudinal control was identical in both parameter sets and the glide slope could be very well followed (results are not shown here). During the four approaches, both localizer and glide-slope deviations could easily be controlled with less than a half dot precision. In figure 6 , the + symbols show the point where the go-around was initiated and the $\times$ symbols where the flight path angle became positive.

Comments of pilots and flight engineers made in the flight test report were that "the airplane can very well be flown with careful inputs", "desired heading can be exactly controlled", "during height changes maximal overshoot was $100 \mathrm{ft}$ ", and "there is no concern about pushing the experiment until landing on a long and wide runway in calm air and without lateral wind". Besides, during the flight and the post-analysis, it appeared that dynamics of the engines at very high thrust were 
significantly faster than predicted by the model used. This lead to a limit cycle in the lateral control (about $+/-3^{\circ}$ of $\beta$ ) loop during max climb maneuvers. After having analyzed the flight-test data, the correction of this limit cycle was straightforward by adjusting the gains on the inner loops at high $\mathrm{N} 1$ values. As low N1 values are required to descent, this gain modification has no consequence on handling qualities during approach and landing.

\section{Conclusion}

To conclude, a simple structure for a propulsion-based control law has been proposed. Simulator as well as flight tests have shown that performance obtained with this structured control law with a low-gain tuning is clearly sufficient for the purpose of landing. Further tests are required and planned in the next months which includes actual landings with this system. Some of the typical autopilot modes are being developed as outer loops generating the references $\phi_{\text {ref }}$ and $\gamma_{\text {ref }}$ given as input of the control law that is presented in this paper. In particular an autoland function is currently under development.The evaluation of the pros and cons of each mode depending on damage cases while taking into account the variability of pilot behaviors and weather conditions will be performed in the near future.

This work has been applied to the VFW-614 ATTAS airplane in both flight tests and simulator tests. Within the next months, it will also be adapted and pursued using two simulators of the DLR Institute of Flight Systems: the Airbus A320 ATRA (Advanced Technology Research Aircraft) simulator and the "Future Military Transport Aircraft" simulator.

\section{References}

1. Frank W. Burcham Jr., John J. Burken, Trindel A. Maine, and C. Gordon Fullerton: Development and Flight Test of an Emergency Flight Control System Using Only Engine Thrust on an MD-11 Transport Airplane. Technical Paper: NASA/TP-97-206217. NASA Dryden.

2. Frank W. Burcham Jr., Trindel A. Maine, C. Gordon Fullerton, and Lannie Dean Webb: Development and Flight Evaluation of an Emergency Digital Flight Control System Using Only Engine Thrust on an F-15 Airplane. Technical Paper: NASA/TP-3627. NASA Dryden. 1997.

3. J. Bull, R. Mah, G. Hardy, B. Sullivan, J. Jones, D. Williams, P. Soukup, J. Winters: Piloted Simulation Tests of Propulsion Control as Backup to Loss of Primary Flight Control for a B747-400 Jet Transport. Technical Memorandum: NASA TM-112191. NASA Ames.

4. Fabio de Almeida: Waypoint Navigation Using Constrained Infinite Horizon Model Predictive Control. AIAA-2008-6462. AIAA Guidance, Navigation and Control Conference and Exhibit, Honolulu, Hawaii, USA, Aug. 18-21, 2008.

5. Fabio de Almeida and Dirk Leißling: Fault-Tolerant Model Predictive Control with Flight Test Results on ATTAS. AIAA-2009-5621. AIAA Guidance, Navigation, and Control Conference, Chicago, Illinois, USA, Aug. 10-13, 2009.

6. Fabio de Almeida and Dirk Leißling: Fault-Tolerant Model Predictive Control with FlightTest Results. Journal of Guidance, Control, and Dynamics, 2010 0731-5090, Vol. 33 No. 2. 